(Aus dem Institut für Pharmakologie und physiologische Chemie zu Rostock.)

\title{
Ueber Glycerinphosphorsäure.
}

\author{
Von
}

\section{Dr. K. Bülow.}

Die Glycerinphosphorsäure wurde zuerst von Sotnits c h e ws$\mathrm{ki}^{1}$ ) als normaler Bestandtheil des Harns nachgewiesen, und ferner baben $R$. Lépi n e und E y m on n e $\mathrm{t}^{2}$ ) quantitative Bestimmungen der gepaarten Phosphorsäure im Urin ausgeführt.

Um das Verbalten der Glycerinphosphorsäure im Organismus zu prïfen und Bedingungen zu finden, unter denen die Menge der Aetherphosphorsäuren überhaupt im Körper vermehrt wird, veranlasste mich Herr Prof. $\mathrm{O}$. $\mathrm{Nasse}$, die folgende kleine Arbeit auszuführen.

Als Versuchsthier diente ein grosser, gut dressirter Hund, der während der Versuchsdaner sein Futter, aus reiner Fleischnahrung bestehend, täglich zur gleichen Stunde erhielt. Der Harn wurde sorgfältig gesammelt.

Die Methode zur Bestimmung der Glycerinphosphorsäure, die allerdings nur eine Bestimmung der Gesammtmenge der Aetherphosphorsäuren darstellt, war folgende.

Um zunächst die Phosphorsäure zu entfernen, wurde dem Harn auf $10 \mathrm{ccm}$ je $1 \mathrm{ccm}$ Magnesiamischnng ${ }^{3}$ ) und dann allmählich $1 / 8$ des Gesammtrolumens an Ammoniak zugefügt. Nach 24-stündigem Stehen wurde der Niederschlag abfiltrirt und wenig: mit verdïnntem Ammoniak (1:3) ausgewaschen.

Das Filtrat wurde, um es von dem ïberscbüssigen Ammoniak zu befreien, eingedampft, wieder mit Wasser verdünnt, neutralisirt und zur Zersetzung der Glycerinphosphorsäure mit $150 \mathrm{ccm}$. conc.

1) Zeitschr. f. physiol. Chemie. 4, 214.

2) Compt. rend. soc. biol. 1882, 622-625.

3) Dieselbe war bereitet nach der Angabe von Fresenius, Anleit. z. quant. chem. Analyse. 6. Autl. pag. 403. Anmerk.: $10 \mathrm{ccm}$ der Mischung fällten $0,24 \mathrm{gr}$ wasserfreie Phosphorsäure. 
Salzsäure fast zur'Trockne gebracht. Darauf wurde der Rückstand. mit möglichst wenig heissem Wasser aufgenommen. Die entstehende Lösung wurde filtrirt, auf $500 \mathrm{cem}$ gebracht und dann mit $50 \mathrm{ccm}$ der Magnesiamischung und mit $225 \mathrm{ccm}$ Ammoniak versetzt. Nach 48 Stunden wurde der gebildete Niederschlag abfiltrirt und nach geringem Auswaschen mit verdünntem Ammoniak in heisser verdiunnter Salzsäure gelöst. Die Lösung wurde, wenn nöthig, filtrirt, auf $50 \mathrm{ccm}$ eingeengt, stark ammoniakalisch gemacht und endlich mit $10 \mathrm{cem}$ Magnesiamischung versetzt. Nach 24 Stunden wurde der gebildete Niederschlag von Ammonium-Magnesiumphosphat abfiltrirt, bis zum Verschwinden der Chlorreaction ausgewaschen und nach dem Veraschen des Filters als: Magnesiumpyrophosphat gewogen.

Nach dem Wiegen wurde der Rückstand in verdünnter Salpetersäure gelöst, in eine salpetersaure Lösung von Ammoniummolybdat eingegossen und erhitzt. Es trat in allen Fällen ein starker Niederschlag von gelber Molybdänphosphorsäure auf.

Nun ist allerdings nicht zu übersehen, dass diese Methode keine ganz exacten Resultate gibt. Das Ammonium-Magnesiumphosphat ist ja in ammoniakalischen Lösungen nicht vollkommen unlöslich, und bei der ersten Fällung in Lösung gebliebene Phosphorsäure wird nach der Concentration zum Theil mit als Glycerinphosphorsäure gefällt und bestimnt werden.

Dieser Fehler ist aber einerseits nicht sehr gross, andererseits baftet derselbe allen Versuchen gleichmässig an, da immer genau mit denselben Mengenverhältnissen gearbeitet wurde. Die Resultate, die in nebenstehender Tabelle zusammengestellt sind, sind also zu Vergleichen wohl geeignet.

Versuch 1 bringt eine Bestimmung der Aetherphosphorsäuren in normalem Harn. Da nur wenig zu erwarten war, wurde die Harnmenge von 5 Tagen verarbeitet. Ich erhielt so aus $8850 \mathrm{ccm}$ Harn $0,0351 \mathrm{gr}_{\mathrm{Mg}_{2}} \mathrm{P}_{2} \mathrm{O}_{7}=0,03098 \mathrm{gr} \mathrm{H}_{3} \mathrm{PO}_{4}$ in Form von Aetherphosphorsäuren, was einer täglichen Ausscheidung von $0,00619 \mathrm{gr}$ entspricht. Dann wurde weiter das Verhalten der Glycerinphosphorsäure im Organismus untersucht. (Versuch 2 und 3.)

Zu diesem Zweck stellte ich mir nach der Vorschrift von $\mathrm{Pel} \circ \mathrm{uze}^{1}$ ) das glycerinphosphorsaure Calcium her.

1) Journ. f. pract. Chem. 36. 257. 


\begin{tabular}{|c|c|c|c|c|}
\hline $\begin{array}{l}\text { Ver- } \\
\text { such }\end{array}$ & $\begin{array}{l}\text { Harn- } \\
\text { menge }\end{array}$ & $\mathrm{Mg}_{2} \mathrm{P}_{2} \mathrm{O}_{7}$ & $\begin{array}{c}\mathrm{H}_{3} \mathrm{PO}_{4} \text { in } \\
\text { Form von } \\
\text { Aetherphos. } \\
\text { phorsäuren }\end{array}$ & \\
\hline $\begin{array}{l}1 \\
2 \\
3 \\
4 \\
5 \\
6\end{array}$ & $\begin{array}{l}1770 \\
1470 \\
1470 \\
1430 \\
1750 \\
1596\end{array}$ & $\begin{array}{l}0,0070 \\
0,0123 \\
0,0135 \\
0,0071 \\
0,0065 \\
0,0071\end{array}$ & $\begin{array}{l}0,00619 \\
0,01085 \\
0,01191 \\
0,00626 \\
0,00573 \\
0,00626\end{array}$ & $\begin{array}{l}\text { Normaltag, Mittel aus } 5 \text { Tagen. } \\
3 \text { gr Glycerinphosphorsaurer Kalk. } \\
\text { I. Tag }\} \text { ca. } 3 \text { gr Glycerinphosphor- } \\
\text { II. Tag saures Natrium subcutan. } \\
3 \text { gr Salol. } \\
3 \text { gr Salol, Mittel aus } 5 \text { Tagen. }\end{array}$ \\
\hline
\end{tabular}

$30 \mathrm{gr}$ Glycerin wurden mit $30 \mathrm{gr}$ an der Luft zerflossenem Phosphorsäureanhydrid vermischt, und die Masse längere Zeit auf dem Wasserbade erhitzt. Nach dem Erkalten wurde mit Wasser verdünnt und darauf Calciumcarbonat eingetragen, bis ein Aufbrausen nicht mehr erfolgte. Dann wurde rom Calciumphosphat und ungelöst gebliebenem Carbonat abfiltrirt und die noch saner reagirende Flïssigkeit mit frisch bereitetem Kalkwasser vollständig neutralisirt. Der sich hierbei ausșcheidende geringe Niederschlag wurde abfiltrirt, das Filtrat eingedampft und nach dem Erkalten das glycerinphosphorsaure Calcium mit Alkohol ausgefällt.

$0,1955 \mathrm{gr}$ dieses Köpers ergaben 0,1217 $\mathrm{gr} \mathrm{CaSO}_{4}$

$$
\text { Ber. für } \mathrm{C}_{3} \mathrm{H}_{7} \mathrm{PO}_{6} \mathrm{Ca} \text {. Gef. }
$$
Ca.
$19,04 \%$
$18,30 \%$.

Es liegt hier also wobl wesentlich neutrales glycerinphosphorsaures Calcium, verunreinigt durch geringe Mengen des sauren Salzes vor. Von diesem Präparat erhielt der Hund 3 gr (Versuch 2), innig gemengt mit 2 gr Calciumcarbonat, um einer sofortigen Zersetzung der Glycerinphosphorsäure durch die Salzsäure des Magens möglichst vorzubeugen. Die Bestimmung der Glycerinphosphorsäure im Harn wurde in derselben Weise ausgeführt, wie oben angegeben, vur wurde zur ersten Ausfällung der Phosphorsäure, um dieselbe vollständig za erreichen, die doppelte Menge Magnesiamischung verwandt. Sie ergab, wie aus der Tabelle ersichtlich ist, nur eine geringe Vermehrung der Glycerinphosphorsäure. Der grösste Theil der Säure ist also zersetzt, und nur eine geringe Menge derselben hat unzerstört den Organismus verlassen.

Ferner wurde auch die Glycerinphosphorsäure subcutan gegeben. Da das Calciumsalz zu schwer löslich ist, so wurde das Natriumsalz dargestellt: $3 \mathrm{gr}$ glycerinphosphorsaures Calcium wur- 
den in Wasser gelöst und mit einer wässrigen Lösung von $1,5 \mathrm{gr}$ Natriumcarbonat versetzt. Nach dem Absetzen wurde filtrirt und das auf ea. $10 \mathrm{ccm}$ eingeengte Filtrat dem Hunde unter die Haut gespritzt (Versuch 3). Das Befinden blieb unverändert, jedoch auch hier war die Zanahme an Glycerinphosphorsäure im Harn nur eine geringe. Am Tage darauf (Versuch 4) war die Menge der Glycerinphosphorsäure im Harn wieder auf den normalen Gehalt zurïckgegangen.

Anhangsweise wurde auch noch der Versuch angestellt, ob sich die Phosphorsäure analog wie die Schwefelsänre mit aromatischen phenolartigen Substanzen zu Aetherphosphorsäuren zusammenlege. Es wurde zu diesem Zweck Salol $\mathrm{C}_{6} \mathrm{H}_{4} \mathrm{COOCO}_{6} \mathrm{H}_{5}$ gefüttert, das bis zu $3 \mathrm{gr}$ pro die, in 2 Portionen zu $1,5 \mathrm{gr}$ Morgens und Nachmittags gegeben, ganz gut vertragen wurde. Nach grösseren Dosen trat Erbrechen ein. Der Harn zeigte starke Phenolreaction, doch war das Resultat, wie aus der Tabelle ersichtlich ist, negativ. Weder in einer einfachen Tagesportion (Versuch 5), noch in der Harnmenge von 5 Tagen (Versuch 6) wurde die täglich ausgeschiedene Menge der Aetherphosphorsäuren vermehrt gefunden.

Aus diesen Versuchen gebt also bervor, dass die Glycerinphosphorsäure, gleichviel, ob sie aus der Nahrung oder aus dem Organismus selbst stammt, im Körper nahezu vollständig zerlegt wird. Die eingeführte Menge des Calciunglycerinphosphats, entsprechend $11,52 \mathrm{gr}$ Distearinlecithin, uibertrifft bei weitem die täglich mit der Nahrung zugeführte Menge von Lecithinen und trotzdem verlässt nur eine ganz geringe Menge Glycerinphosphorsäure unzersetzt den Organismus. Es ist also wahrscheinlich, dass die aus den Lecithinen, sei es im Darmcanal, sei es in den Geweben, freiwerdende Glycerinphosphorsäure vollständig weiter zerlegt und als Phosphorsäure ausgeschieden wird. Dies ist um so einleuchtender, da nach Versuch 5 und 6 die Phosphorsäure nicht die Fähigkeit zu besitzen scheint, sich im Körper analog der Schwefelsäure mit phenolartigen Substanzen zu Aetherphosphorsäuren zu vereinigen. 